\title{
Urgences
}

\section{La stratégie du conteur masqué}

\section{Guy Gauthier}

Numéro 32, mai 1991

Lectures de bandes dessinées

URI : https://id.erudit.org/iderudit/025647ar

DOI : https://doi.org/10.7202/025647ar

Aller au sommaire du numéro

Éditeur(s)

Urgences

ISSN

0226-9554 (imprimé)

1927-3924 (numérique)

Découvrir la revue

Citer cet article

Gauthier, G. (1991). La stratégie du conteur masqué. Urgences, (32), 44-58.

https://doi.org/10.7202/025647ar d'utilisation que vous pouvez consulter en ligne.

https://apropos.erudit.org/fr/usagers/politique-dutilisation/ 


\section{La stratégie du conteur masqué Guy Gauthier}

\section{Un récit d'aventures}

Le passage qui va faire l'objet de quelques remarques portant sur la construction du récit est extrait du tome IV des Passagers du vent, de François Bourgeon (L'heure du serpent, planches 33 à 40) 1 .

Nous sommes sur la côte occidentale de l'Afrique, en 1781. Le navire négrier La Marie-Caroline, venu d'Europe, fait escale le temps d'embarquer un chargement d'esclaves à destination des Caraibes. Les aventures des personnages principaux (présents dans les cinq volumes de la somme romanesque-dessinée) font l'objet de la trame narrative de l'album.

La "séquence" (cette appellation est approchée, puisqu'elle fait référence au cinéma) prise en compte met en scène plusieurs personnages en des lieux précisément localisés ou situés sur un itinéraire. Tout se passe entre Abomey, capitale du royaume de Kpëngle, Juda, village du même royaume, Fort-Saint-Louis, comptoir français de traite des Noirs, et Fort-William, comptoir anglais, même spécialité. Un segment narratif (partie de séquence dont les vignettes s'enchaînent de façon linéaire), enchâssé dans le récit sans interférer sur son déroulement, a pour cadre le village lacustre de Juda. Il prolonge les planches 28 et 29 .

Pour ne pas revenir sur les caractéristiques des personnages dont l'identification est indispensable pour la compréhension du récit, voici une brève présentation de chacun d'entre eux par ordre d'entrée en scène:

Mary, jeune Anglaise de mœurs très libres, passagère de la Marie-Caroline, mère d'Enora.

François Vignebelle, très jeune officier de la Marie. Caroline.

Viaroux, subordonné de Montaguère à Fort-Saint-Louis, a tenté de séduire Isa (voir plus loin), et empoisonné son amant

1 François Bourgeon, L'heure du serpent, coll. "Les passagers du vent", tome IV, Grenoble, Glémal, 1982. 
Hoel. Mis aux arrêts pour une nouvelle tentative, il s'est échappé d'Abomey et se dirige vers Fort-Saint-Louis.

John Smolett, officier anglais devenu fou, ancien amant de Mary, et père d'Enora. Il s'est échappé de Fort-William et se dirige vers Fort-Saint-Louis.

Isa, principale héroïne de l'ensemble de la saga romanesque, jeune femme intrépide qui lutte pour la survie de son amant Hoel et espère en la sorcellerie. Elle n'a pas de rôle actif dans le drame relaté par la séquence.

Le jeu des rencontres et des séparations des personnages articule entre eux les différents segments narratifs. Mary constitue le personnage central tout au long de la séquence; sa présence, même comme simple spectatrice suffit pour identifier le segment narratif principal. Voici, sur cette base, une proposition de classement des segments.

Le segment principal commence dès le début de la planche 30 , s'interrompt à l'avant-dernière vignette de la même planche, et se poursuit dans les quatre dernières vignettes de la planche 31 . La toute dernière, cependant, voit surgir un personnage jusque là hors présence (John): elle constitue un "moment de risque" et une rupture. La seconde partie du segment considéré commence donc avec la dernière vignette de la planche 31, et se poursuit avec les sept premières vignettes de la planche 32 . Le lieu est identique (intérieur de case éclairé), mais les personnages sont désormais quatre (Mary, François, la petite Enora, John).

La troisième partie du segment se poursuit, après une courte transition explicative d'une seule vignette, avec les mêmes personnages jusqu'à la fin de la planche 32 . Ayant quitté Fort-Saint-Louis sous la menace de John, ils marchent la nuit dans la savane.

Nous les retrouvons à la planche 33 , à l'exception des trois premières vignettes consacrées à une action parallèle. Ils sont désormais au bord d'un marécage. François n'est plus que spectateur impuissant de la confrontation Mary-John, à son paroxysme. Cette confrontation se poursuit planches 34 et 35 . Toutefois, la planche 35 amorce un segment narratif dérivé, puisque François s'éloigne du groupe pour sauver Enora, précipitée dans le marais par son père. La dernière vignette de cette même planche 35 appartient à un segment narratif parallèle. 
46

La planche 36 est, du point de vue narratif, une mosaïque. Seules les vignettes 1 et 4 (superposées dans la mise en page) continuent le segment «Mary-John», qui se poursuit avec les quatre premières vignettes de la planche 37. Quant à son contenu narratif, on peut diviser cette troisième partie en deux, puisque le geste meurtrier de John à l'égard d'Enora laisse les deux amants face à face, éliminant deux personnages secondaires.

On les retrouve à la planche 38 , mais l'intrusion d'un nouveau personnage, jusque-là en segment parallèle, constitue un nouveau «moment de risque", et l'amorce d'une véritable quatrième partie. Pendant les quatre premières vignettes, l'intrusion est seulement sonore (un coup de feu), mais l'arrivée effective de Viaroux fait de Mary une spectatrice du duel qui s'engage entre les deux hommes. Ce duel dure toute la planche 39 , puis la planche 40 , exception faite des deux dernières vignettes panoramiques (avec une incrustation) qui marquent la fin des grandes peurs de la nuit.

Résumons ce très long segment narratif

1 - Fort-Saint-Louis, une case: Mary et François, scène d'amour (pl. 30-31, 12 vignettes);

2 Même lieu: arrivée et menaces de John (pl. 31-32, 8 vignettes);

3 - Départ clandestin et marche vers le marais: affrontement violent entre Mary et John (pl. 32-37, 37 vignettes, en comptant à part l'amorce du segment dérivé François-Enora);

4 Arrivée de Viaroux, et duel dans le marais (pl. 38-40, 37 vignettes).

Il reste à signaler trois segments narratifs parallèles, deux en amont du précédent (l'un centré sur Viaroux, l'autre sur Smolett), un en aval (ou en dérivation). Les points de convergence des segments Smolett et Mary, puis Viaroux et Mary-Smolett constituent les temps forts du récit (les "noyaux" selon Roland Barthes), le point de divergence du segment François-Enora n'a qu'une fonction secondaire (catalyse selon $R$. B.). Cependant, le point de convergence du segment Viaroux et du segment François constitue également un "moment de risque ", donc un " noyau". 
Segment Viaroux: pl. 30, 31, 33, 35, 36, 37, 8 vignettes;

Segment Smolett: pl. 31, 7 vignettes;

Segment François : pl. 35-37, 9 vignettes.

Le schéma suivant fournit quelques repères sur l'imbrication des segments:

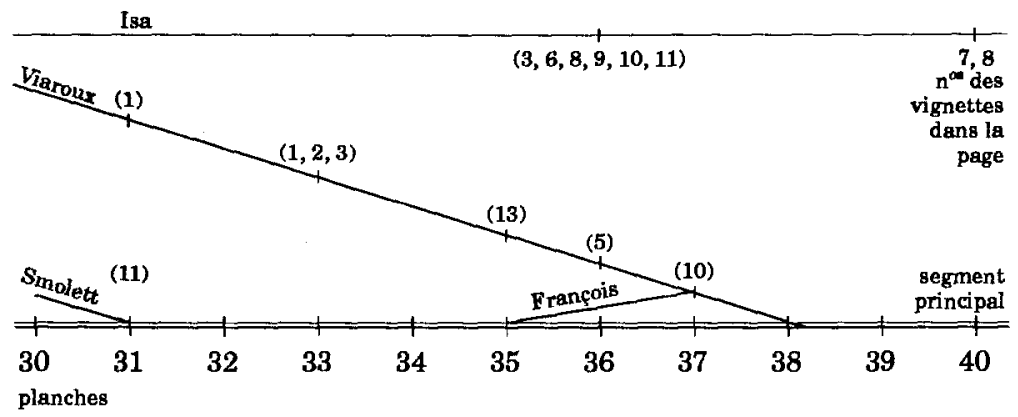

\section{La dialectique du narratif et du visuel}

Le récit en vignettes s'organise dans l'espace de la page, et c'est le lecteur qui réintroduit le temps et l'intelligibilité de la narration. On peut concevoir - ce qui n'est pas rare en bande dessinée - une lecture strictement plastique, qui sacrifie l'intelligibilité de l'enchaînement à la contemplation esthétique de la planche.

Il s'agit cependant d'un récit romanesque, qui prend le relais des romans d'aventures maritimes et coloniales en honneur jusque dans les années trente. Le lecteur est bien invité à lire, ce à quoi l'aident les mots écrits qui remplacent les dialogues du roman. Pour le reste, il doit se fier à l'enchaînement des faits. Pour le repérer, il dispose des éléments descriptifs et signalétiques mis en évidence à son intention (distinction des personnages par quelques traits pertinents, reconnaissance des lieux).

La juxtaposition des vignettes lui permet dès lors de déceler les liens de consécution et de conséquence qui attestent de la cohérence du récit.

Certaines bandes dessinées insistent sur cet aspect en disposant les vignettes de façon régulière, facilitant une 
lecture lisse, "cinématographique". Le regard passe d'une vignette à l'autre sans heurts, même si le passage du plan d'ensemble au gros plan, ou de la plongée à la vision horizontale, introduit un dynamisme absent des bandes dessinées «primitives» de la fin du XIX ${ }^{\mathbf{e}}$ siècle.

L'ensemble retenu l'a été justement parce que le récit bénéficie de figures plus spécifiques de la bande dessinée, qui jouent sur les procédés plastiques en les mettant au service d'une grande mobilité, d'une gymnastique de l'agencement qui rapproche des conditions du spectacle (qu'on pense aux montreurs d'images des spectacles forains qui variaient à volonté le rythme de passage de leurs tableaux mobiles).

Deux de ces procédés retiendront notre attention: les variations colorées et les «incrustations", images incluses dans d'autres images.

\section{Les ruses du spectre}

Chaque segment et, pour le plus long d'entre eux, chaque partie, comportent une dominante colorée que ne justifient que partiellement les contraintes réalistes. On peut néanmoins considérer comme telles celles qui permettent de distinguer l'obscurité de la nuit dans la savane de l'intérieur de la case éclairée. Encore faut-il s'entendre sur le "réalisme": la nuit est bien une réalité référentielle objective, mais la traduction colorée obéit à des conventions, puisque l'image de la nuit se devrait d'être entièrement noire, ce qui serait absurbe. Le cinéma connaît bien cette difficulté, et la contourne par l'éclairage (nuit américaine). La bande dessinée a d'autres possibilités.

Si on oublie cette restriction, on peut suivre chaque segment indépendamment de la logique narrative et du contenu. Outre les noirs et les bruns, indispensables pour renforcer l'impression de nuit, chaque personnage - et, partant, le segment dont il est la raison d'être - évolue sous le signe d'une dominante colorée. Qu'est-ce qu'une dominante? Une couleur qui "accroche" le regard, dont la mémorisation demande quelques fractions de seconde, au point que c'est cellelà qu'on retient lorsqu'une vignette séparée est présentée brièvement à un sujet qui ignore tout du contexte. Ce n'est 
pas forcément une question de surface. À vrai dire l'explication de ce phénomène relève de l'étude de la vision, mais la narration par l'image introduit un jeu de contrastes et d'oppositions qui oblige à prendre en compte, comme unité minimale visuelle, non pas la vignette, mais un ensemble de vignettes, parfois une page entière (ou une double page) qui coïncide très souvent avec le "segment narratif » tel qu'il a été défini plus haut. Nous avons donc affaire, comme souvent dans les études iconiques, à un phénomène d'influences réciproques entre le sens et la perception pure, sans qu'on décèle immédiatement ce qui l'emporte de la construction "primaire" qu'opère le sujet par le jeu du mécanisme de la vision envisagé comme neutre (ce qu'il est rarement), et la construction "secondaire" qui résulte de l'apport de l'imaginaire, de la culture, ou de la recherche du sens.

Ces précautions prises, on note que les dominantes dans la séquence qui nous intéresse, se répartissent de la façon suivante dans le spectre (violet-indigo-bleu-vert-jauneorangé-rouge). Les couleurs primaires présentent ellesmêmes toute une gamme, elles sont plus difficiles à identifier quand on passe de l'une à l'autre, et sont modifiées par l'adjonction de gris, ce qui atténue les distinctions.

Rappelons que nous avons distingué cinq segments, définis par le ou les personnages qui donnent à chacun son unité:

1 - Mary-François,

2 - John.

Ces deux segments n'en font plus qu'un avec l'entrée de John dans la case:

3 - Mary-John (François n'est plus qu'un comparse),

3a - François (en dérivation),

4 - Viaroux.

Viaroux rencontre d'abord François (qui a retiré Enora du marais), puis John et Mary, ce qui amorce le segment 5 (Viaroux-John-Mary). dérée.

Le segment 6 (Isa) n'interfere pas sur la séquence consi- 
50

Dans l'ordre du spectre, on note les dominantes suivantes:

Violet-indigo-bleu: Viaroux;

Bleu-vert-jaune : John;

Jaune-orangé: Mary;

Orangé-rouge: Isa.

À chaque personnage est ainsi affecté un indicatif coloré. Il est tentant évidemment de transposer cet indicatif en thème à portée symbolique. Que Johṇ Smolett évolue dans des couleurs froides convient à sa folie, de même que la généreuse Mary est comme auréolée par l'intensité lumineuse des jaunes et des roux qui résistent même à la nuit. Cependant, ce symbolisme de convention n'est pas l'essentiel. Par contre, la lecture des niveaux narratifs en est facilitée, ce qui conduit à analyser cette distribution des couleurs comme un acte d'énonciation destiné à piloter la narration, ce qu'illustrent les quelques exemples suivants.

\section{Les trois premières vignettes de la planche 31}

La première montre Viaroux de face, cadré à mi-corps. La seconde montre Smolett, également de face, mais cadré en gros plan. La juxtaposition serait identique si les deux vignettes opéraient un effet de montage classique par changement d'échelle d'un même personnage représenté selon le même axe. Un élément de contenu permet d'écarter cette hypothèse: le chapeau. Mais l'élément décisif pour indiquer qu'il y a changement de segment, et pas seulement changement de plan à l'intérieur d'un même segment, c'est justement la rupture introduite par les couleurs: on passe de la "nuit de Viaroux" (qui est aussi la nuit dans la savane) à la "nuit de Smolett", un autre climat, psychologique et non plus seulement descriptif. La vignette suivante prend du recul et montre Smolett toujours de face, mais cadré à mi-jambe, découvrant en arrière-plan un paysage nocturne qui contraste (notamment par la couleur du ciel) avec le paysage qu'on devine autour de Viaroux. 


\section{Les six dernières vignettes de la planche 33}

L'affrontement de Mary et de Smolett commence véritablement ici. Le dialogue suffirait pour en rendre compte, mais ce sont les oppositions colorées qui retiendront notre attention. Si le plan de très grand ensemble qui introduit le paysage du marais, cadre du combat à venir entre Viaroux et Smolett, est placé sous l'indicatif de Smolett, la vignette en incrustation dans le paysage (nous reviendrons sur le procédé) réintroduit (bien que ce soit la même nuit et qu'il n'y ait aucune variation attestée d'éclairage) l'indicatif de Mary, avec une simple atténuation du roux de sa chevelure. La bande de quatre vignettes situées juste au-dessous est une sorte de présentation du conflit par le simple jeu et contraste des couleurs ( 1 - face-à-face; 2 - contre-attaque de Mary; 3 - concession à la folie de Smolett; $4 \cdot$ opposition maximale). La couleur cependant ne fait qu'exécuter sa partition: situations, dialogues et cadrages jouent la leur. Ce sont le cadrage et les variations de couleurs qui contribuent essentiellement au passage du dramatique à l'épique, de la relation conduite par l'événement à l'histoire qu'on raconte. En réintroduisant le conteur, même impersonnel (nous sommes loin en effet des vignettes du conteur en gros plan qui ponctuent platement d'anciennes bandes dessinées comme Les histoires de l'Oncle Paul), la bande dessinée d'aventures retrouve sa fonction la plus productive. Tous les arts du récit ont d'ailleurs tenté de réintroduire sans le mettre en scène ce personnage essentiel du conte qu'est le conteur. Brecht recommandait aux comédiens de s'inspirer du "théâtre qui se joue dans la rue", quand le témoin mime l'accident, ou le locataire son propriétaire (Du théâtre quotidien).

\section{La planche 36}

Cette planche est particulièrement significative, et servira de dernier exemple. La première moitié supérieure se compose de six vignettes, inhabituellement régulières, et de format carré. La seconde moitié, sur laquelle nous aurons l'occasion de revenir plus loin, est faite d'une grande vignette (paysage nocturne, avec case sur pilotis au milieu d'un marécage), avec quatre vignettes de format inégal s'agençant en incrustation comme une mosaïque incomplète. 
52

Isolée, cette page est illisible comme récit ou fragment de récit. Il n'y a pas deux vignettes consécutives qui se rapportent au même segment. Les vignettes 1 et 4 sont à rapporter au segment 3 (affrontement Mary-John); la vignette 2 s'insère dans le segment dérivé 3a (sauvetage d'Enora par François); la vignette 5 dans le segment 4 (Viaroux). Toutes les autres font partie du segment 6 (Isa), étranger à la séquence considérée.

À considérer simplement les dominantes colorées, les six vignettes supérieures se distinguent en deux séries de trois formant deux équerres parfaitement emboîtées. La série de gauche est dominée par l'indicatif Smolett tempéré par lindicatif Mary qui tente de séduire et de calmer le fou. La série de droite associe Viaroux et Isa dans les teintes plus chaudes de la nuit tropicale, ce qu'elle serait partout sans l'influence maléfique de la folie de Smolett. On retrouve cette dominante dans la vignette-paysage de la partie inférieure. Par contre, les incrustations, en dominantes rouges et ocres évoquent comme les éclats sonores d'une scène de sorcellerie - ou peut-être s'agit-il simplement du rêve d'Isa. Toute la planche se présente donc comme un récit haché, syncopé, associant sans lien logique apparent des segments dont le lecteur n'a pu apprécier encore la cohérence qui ne deviendra sensible que plus en aval. Là encore, le conte se dissimule derrière le conteur, ou pour le dire autrement, l'énoncé derrière l'énonciation.

\section{La fonction énonciative de l'incrustation}

L'incrustation consiste à enchâsser une vignette dans une autre plus grande, ou variante, à cheval sur deux vignettes. Le procédé est couramment employê en vidéo. Il l'a été au cinéma du temps du muet, il l'est encore dans le cinéma expérimental et peut être rapproché des "écrans seconds" (miroirs, fenêtres, etc.) ${ }^{2}$.

En bande dessinée, il constitue une variante de la juxtaposition, et pourrait être intégré dans les procédés de montage, s'il ne brisait de manière délibérée le «naturel " dans la succession des événements tel que tente de l'accréditer la

2 Voir Christian Metz, L'énonciation interpersonnelle ou le site du film, Paris, Méridiens Klincksieck, 1991, p. 71. 
plate succession des vignettes, quand elles sont de taille identique. C'est que là encore, le conteur reprend sa place au détriment de la * transparence * du récit. En ce sens, l'incrustation, comme la couleur réintroduit l'«épique » au sens où l'entendait Brecht.

Cette fois encore, limitons-nous à deux exemples.

\section{Les vignettes 2 et 8 de la planche 35}

Les deux premières vignettes consécutives de cette planche (figure 1) juxtaposent en un effet classique de montage la lutte corps à corps de John et de Mary (qui tente d'aller au secours de l'enfant précipitée dans le marais) et le visage de la fillette en pleurs émergeant à peine de l'eau.

Le visage de François, en gros plan, mais inscrit dans une très petite vignette, apparaît en incrustation à cheval sur les deux vignettes. C'est, simultanément, l'apparition d'un tiers - on l'avait presque oublié - et une relance de l'action: quelqu'un a vu la scène et va pouvoir sauver l'enfant. Cette apparition, cependant, rompt la continuité narrative «naturelle», d'abord parce que le carré où s'inscrit le visage est un corps étranger, ensuite parce que François, dont on sait bien, évidemment, qu'il regarde la scène, semble regarder le lecteur, avec l'effet d'interpellation que cela comporte. Le film use du procédé avec l'adresse au spectateur, mais le dispositif cinématographique est plus favorable à la confrontation écran-spectateur que le livre, consacré habituellement au déchiffrage de l'écriture. Le regard de François intervient donc comme une déstabilisation de la narration fluide. La vignette suivante enchaîne non pas sur l'affrontement MaryJohn, mais sur l'acte de sauvetage qui a été amorcé par la vignette d'adresse au lecteur. Celui-ci se trouve donc à endosser le rôle supplémentaire de démarrage d'un segment narratif dérivé (celui qui a été arbitrairement noté 3-a). C'est à nouveau, par un coup de force de l'énonciation, le retour du conteur, qui impose sa loi au cours tranquille de la narration événementielle.

On retrouve à la vignette 8 , à nouveau en incrustation, François qui a sauvé Enora et regagne le rivage. Cette vignette a également un double statut: elle continue le segment 3-a, mais elle s'insère dans le segment 3, puisqu'elle correspond explicitement à une vision de Mary, ainsi rassurée sur 

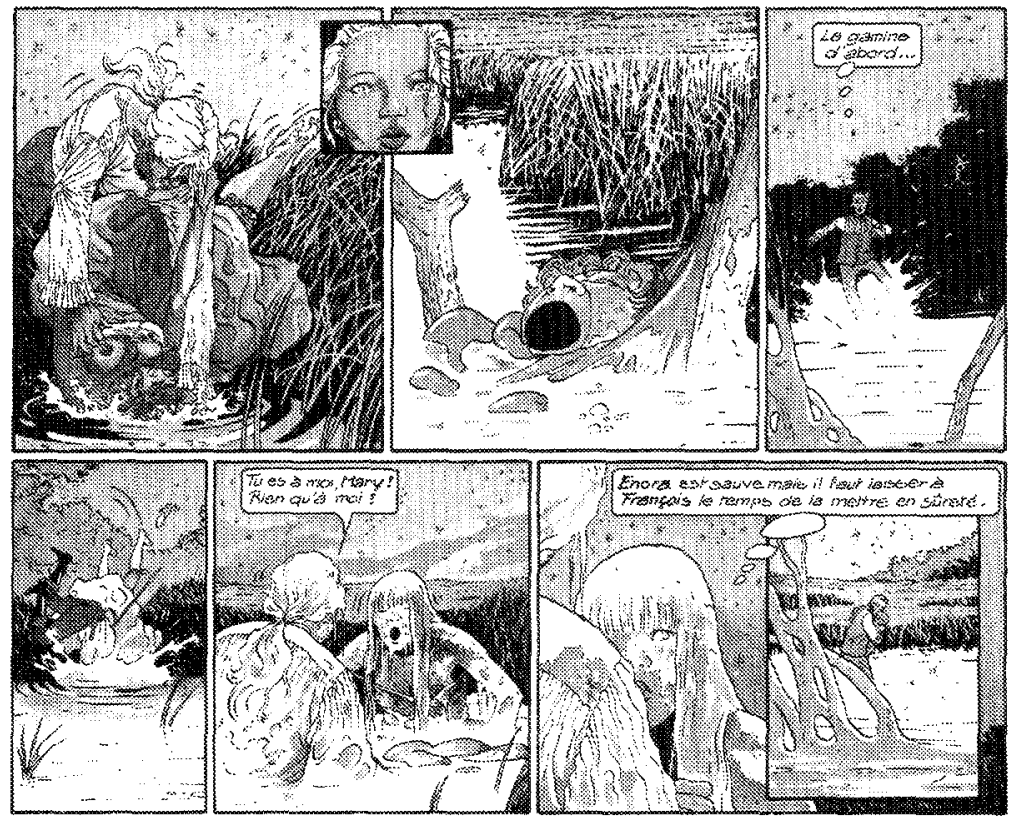

Figure 1 
le sort de son enfant. Image donc à la fois subjective et objective, cette vignette atteste de la capacité du conteur à endosser plusieurs rôles. Une fois de plus, on pense à Brecht, lorsqu'il donne au comédien l'exemple du témoin d'un accident qui mime tour à tour les différents personnages.

Il a déjà été parlé de la fonction à la fois onirique (est-ce le rêve d'Isa?), potentiellement réaliste (est-ce le récit dynamique d'une cérémonie de sorcellerie?) et purement rythmique (les images n'ont-elles que fonction de métaphore sonore?) de l'agencement de la planche 36 . Là encore, il y a quelque part un manipulateur virtuel, qui atteste de la toutepuissance du conteur, maître du récit et brouilleur de transparence.

\section{La planche 39}

Cette page (figure 2) relate une partie du duel ViarouxSmolett, qui commence au bas de la planche 38, et se termine par l'enlisement des deux combattants sur les deux tiers de la planche 40. Remarquons en passant qu'en termes d'action, ce duel représente une unité narrative, alors que dans la classification adoptée par personnages, ce n'est qu'une partie non pertinente du segment narratif principal, qui résulte de la jonction de tous les personnages (Isa exceptée).

La planche 39 se décompose ainsi: les quatre premières vignettes sont centrées sur les personnages (les duellistes en pied, Mary en gros plan); les suivantes montrent l'éloignement des combattants en opposition avec la terreur en gros plan des spectateurs impuissants; la dernière enfin qui occupe en panoramique toute la largeur de la planche peut être considérée comme un "indice" selon Barthes, puisqu'elle ne fait que traduire une atmosphère, celle d'une nuit tropicale mystérieuse et indifférente au drame, les combattants se réduisant à des silhouettes dérisoires.

Dans la première bande, une seule incrustation: celle de Mary vue de face, comme s'adressant au lecteur. On a déjà noté que ce "regard d'adresse" marquait une sophistication du dispositif, et dévoilait la marchinerie du conteur, en rompant la succession "objectivement" narrative des vignettes. Le plan suivant de Mary ( $4^{\mathrm{e}}$ vignette) réintègre cette succession, 

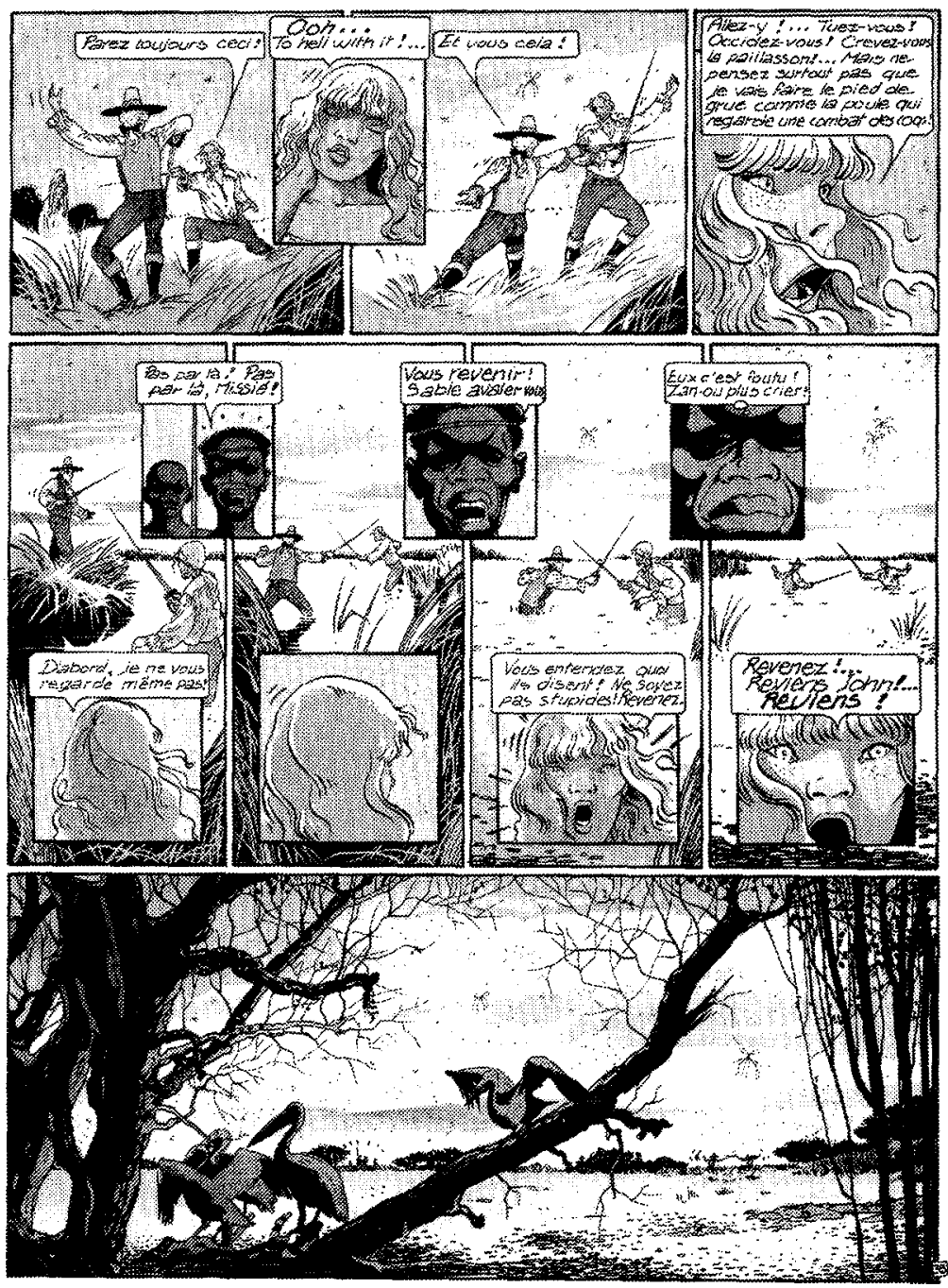

Figure 2 
mais Mary est alors spectatrice (en très gros plan) du combat. Son indicatif coloré l'oppose cependant à celui des combattants (la dominante Viaroux se mêle à la dominante Smolett, le tout éclairci par les signes de l'approche de l'aube) et permet de distinguer nettement les deux plans.

La bande suivante est plus caractéristique du procédé de l'incrustation. En haut, de face, en plans de plus en plus rapprochés, comme un * effet zoom ", deux Noirs (les serviteurs de Viaroux) dont le regard (vers les combattants, mais aussi vers le lecteur) et les paroles inutiles traduisent l'issue inéluctable du combat (les péripéties en sont sans importance, d'où leur traitement). En bas, dans les vignettes du combat, et non plus à cheval comme pour les deux Noirs, Mary, d'abord de dos (elle ne veut pas voir le combat, mais du coup elle impose la présence de sa chevelure rousse), puis de profil, puis deux fois de face en "effet zoom». On retrouve son regard initial (vignette 2) déformé par l'émotion, moins encore que dans la vignette 5 de la planche 40 (figure 3 ) où elle prononce le mot qui clôt la séquence du duel: «Rentrons ». C'est alors qu'en deux vignettes panoramiques, relatives aux deux segments qui n'ont pas fait jonction (Isa et le segment principal), on voit le soleil se lever, mettant fin à cette nuit doublement éprouvante: la mort de Viaroux et de Smolett; le cauchemar libérateur d'Isa.

\section{Derrière le conte, le conteur}

D'autres procédés, en particulier les variations de format, permettent de rompre ainsi la fiction de la succession chronologique des vignettes, autrement dit de la transparence du récit que la bande dessinée d'aventures classiques (années 30) avait tenté d'imposer pour des raisons de légitimité (et parce que telle était en ces années-là l'idéologie narrative dominante du cinéma). Désormais, la machinerie du conteur reprend le dessus, et même au sens strict, puisque les exemples ne sont pas rares, où l'intelligibilité du récit est sacrifiée à la dextérité du conteur. En ce sens, Bourgeon, qui tente d'équilibrer intelligibilité et dextérité, peut être situé en transition entre la bande dessinée d'aventures classique (dont le dernier grand représentant est sans doute Hugo Pratt) et la bande dessinée désireuse d'afficher son dispositif. Littérature, théâtre, cinéma, en sont passés par là. 

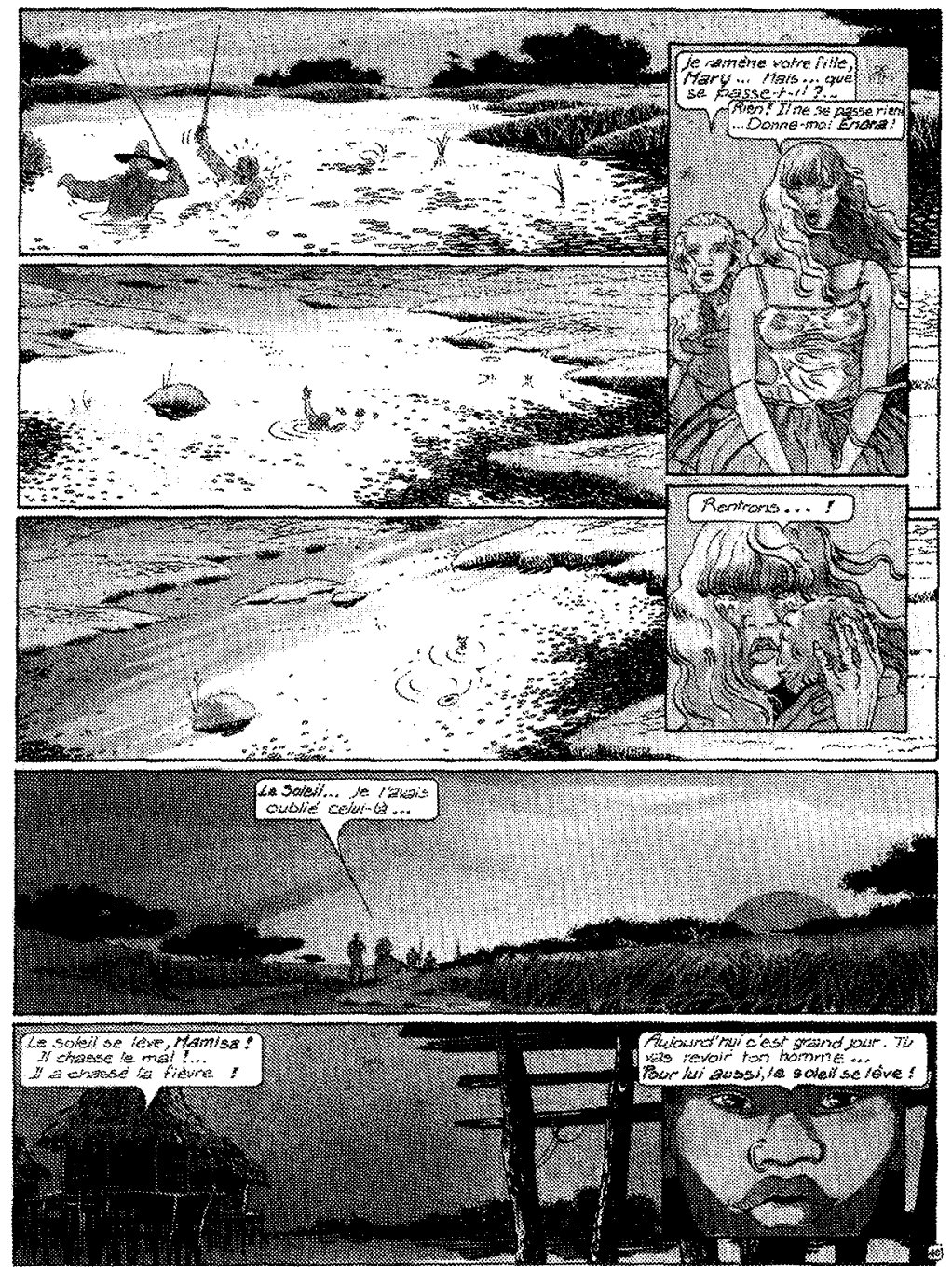

Figure 3 